International Egyptian Journal of Nursing Sciences and Research (IEJNSR)

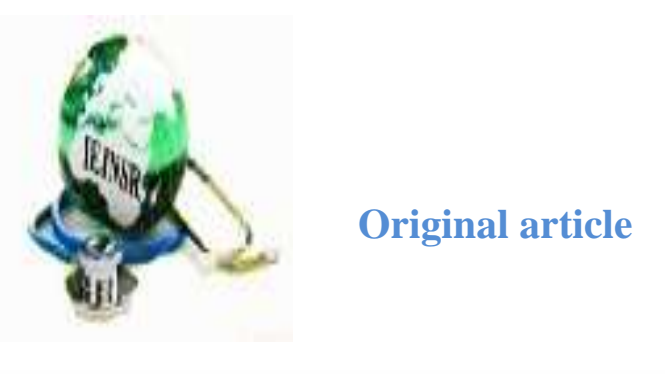

Received 2021-05-10

Accepted 2021-05-28

Published 1- 7-2021

\title{
Cerebral Palsy in pediatrics: Review Article
}

\section{Heba Ibrahem Mohamed \\ Lecturer of pediatric $\mathcal{N}$ ursing, Faculty of $\mathcal{N}$ ursing, Kafre E[ sheikf university, Egypt.}

Corresponding author: hebaibrahe855@yahoo.com

\begin{abstract}
Cerebral palsy (CP) is a neurodevelopmental condition caused by a disturbance in the infantile or fetal brain's development. It affects more than 17 million people all over the world. The aim of this review paper is to include an in-depth review of the literature surrounding the causes, diagnosis, and related disorders of $\mathrm{CP}$, as well as therapeutic approaches that may contribute to a comprehensive treatment plan for children with cerebral palsy Contrary to common opinion, CP can be caused by a variety of factors, including hypoxia or ischemia at birth. The motor function, part(s) of the body affected, and severity must all be determined during diagnosis; diagnosis techniques have recently improved. The associated diseases must also be identified in order to receive medication.
\end{abstract}

\section{Keywords: Cerebral Palsy, Treatment, Intervention, Pediatrics}

\section{INTRODUCTION}

The most common cause of physical disability in children is cerebral palsy (CP). It refers to a set of permanent movement and postural defects that limit function and are caused by non- progressive injuries to the infant brain during development. Cerebral palsy can cause primary or secondary feeling, vision, and cognition problems in addition to motor dysfunction, epilepsy, and musculoskeletal disorders, affect memory and communication, (1) 


\section{Prevalence}

For every 1,000 babies born in the United States, approximately three will be diagnosed with some type of cerebral palsy by the time they are 8 years old. This result makes $\mathrm{CP}$ the most widespread cause of childhood disability. The prevalence of cerebral palsy is around 1.53 per 1000 live births in both developed and developing countries .A study in AlQuseir City, Red Sea Governorate reported a prevalence in children of 3.06 per 1000 live births ${ }^{(2)}$

\section{Causes of CP}

Hypoxia or ischemia at birth was once believed to be the only cause of cerebral palsy. While hypoxia or ischemia at birth is still potential causes of $\mathrm{CP}$, recent research has revealed a plethora of other potential causes and risk factors. It's possible that a child may be born with or develops CP. Cerebral palsy occurs when a condition or a combination of problems causes an injury to a foetal or infantile brain's growth. A new research backs up previous observations that more $\mathrm{CP}$ triggers have a genetic basis. ${ }^{(1,3)}$.Other issues include obstructed blood flow to the brain, which can lead to foetal stroke and blood clotting, neonatal infections, and head injuries that cause brain damage. And after birth, all of these variables placed the child at a higher risk of developing $\mathrm{CP}^{(4)}$

\section{Types of C P:}

Cerebral palsy is classified by the type of movement problems (spastic, diskinetic, ataxia, dystonia ) or by the body parts affected (hemiplegia, diplegia, and quadriplgia) The final classification based on the severity includes gross motor skills, fine motor skills, and communication ${ }^{(4)}$.

\section{Diagnosis of CP}

A mixture of medical methods and instruments, such as term-age magnetic resonance imaging, the Qualitative
Assessment of General Movements, the Hammersmith Infant Neurological Examination, and the Developmental Assessment of Young Children, is the best way to diagnose $\mathrm{CP}$. The patient's medical history may also provide useful knowledge for diagnosis. The specific tests required for each person would differ depending on the patient's age, suspected form of $\mathrm{CP}$, and other external factors such as medical resource availability. A small group of medical and nursing practitioners can perform all of these tests and equipment (5)

\section{Associated Illnesses}

Because of the essence of $\mathrm{CP}$ as a condition, the majority of children with $\mathrm{CP}$ have at least one other disorder. There are several different types of related conditions, as well as different degrees of severity. Children with mild to extreme cerebral palsy have a delayed physical growth and development. As a limb develops, a CP child with spasticity of the muscles will experience a lack of stretching of the muscles and tendons. Contractures may occur as a result of spastic muscles not developing as quickly as the bones, causing issues with both gross and fine motor skills. Patients with such gross motor function issues can be unable to walk without the use of an assistive device. ${ }^{(6)}$

Children that are non-ambulatory tend to have markedly reduced bone mass which can lead to osteopenia, osteoporosis, fracture, scoliosis, or pain. Seizures are another common associated disorder for $\mathrm{CP}$ patients with about one half of pediatric $\mathrm{CP}$ patients experiencing at least one kind of seizure. Some patients with CP may suffer from abnormal sensations and perceptions ${ }^{(7)}$

Impaired oral-motor functions are a popular $\mathrm{CP}$ co-morbidity that can contribute to a slew of other issues. Dysphagia (difficulty swallowing), 
hypoxemia, vomiting, and aspiration pneumonia are all symptoms of gastroesophageal reflux, as well as inadequate nutrition, inability to survive, drooling, and speech problems. Speech and language disorders may also be the result of poor oral-motor control. The diagnosis of $\mathrm{CP}$ is often associated with cognitive impairment and behavioural problems ${ }^{(8)}$

\section{Cerebral Palsy Management}

Since there is no treatment for $\mathrm{CP}$, those who are affected will live with it for the rest of their lives. Children with $\mathrm{CP}$, on the other hand, usually have a team of health care practitioners working with them. A primary care physician, a physical therapist, an occupational therapist, a speech and language pathologist, a psychologist, an orthopaedic surgeon, an ophthalmologist, an audiologist, and/or a special educator and Rehabilitation nurse may all be part of a healthcare team (9)

Physical Therapy is a form of treatment that is used Physical therapy is one of the first treatments prescribed for children with cerebral palsy. Physical therapists (PTs) are health care providers who specialise in movement and use prescribed activities to improve quality of life. Occupational Therapy (OT) is a form of therapy that Occupational therapy is a common technique for children with CP. An occupational therapist (OT) works with children to help them acquire the skills they need to perform everyday tasks. In addition to physical therapy and occupational therapy, children with CP can receive a variety of other medical strategies. Exercise, speech and language pathology, drugs, and surgical procedures are examples of additional treatments ${ }^{(\mathbf{1 0})}$

\section{Impact of CP on both child and family}

Parents of children with cerebral palsy $(\mathrm{CP})$ often experience higher levels of stress than other parents. This is because children with $\mathrm{CP}$ need long-term care. The child can be deprived of educational opportunities, recreational facilities, and opportunities to develop friendships Also, the attitudes and stigmatization of others could be very disturbing to parents (11)

\section{REFERENCES}

1. Graham H et al. ( 2016): Cerebral palsyNat Rev Dis Primers ; 7 ( 2): 15082 .

2. Khalil M, Elweshahy $\mathrm{H}$, Abdelghani H, Omar T . (2018): Quality of care provided to children with cerebral palsy .Alexandria, Egypt; 24(6)

3. Cerebral Palsy Alliance Research Foundation. (2018): What is Cerebral Palsy? Retrieved January 28, 2020, from https://cparf.org/what-is-cerebralpalsy/

4. Gao J, Zhao B, He L, Sun M, Yu X, Wang L. (2017) Risk of cerebral palsy in Chinese children: A N:M matched case control study. J Paediatr Child Health.;53(5):4649.

5. Jystad KP., et al. (2017) :Congenital anomalies and the severity of impairments for cerebral palsy. Dev Med Child Neurol;59(11):1174-80.

6. Fahey M., et al (2017): undefined. The genetic basis of cerebral palsy. Developmental Medicine and Child Neurology; 59(9): 462-469.

7. Chang, E., Ghosh, N., Yanni, D., Lee, S., Alexandru, D., \& Mozaffar, T. (2013):A Review of Spasticity Treatments: Pharmacological and Interventional Approaches. Critical Reviews in Physical and Rehabilitation Medicine;25 (1-2): 11-22. doi: $10.1615 /$ critrevphysrehabilmed.201 3007945 
8. Chen, C.-L., Hong, W.-H., Cheng, H.-Y. K., Liaw, M.-Y., Chung, C.Y., \& Chen, C.-Y. (2012): Muscle strength enhancement following home-based virtual cycling training in ambulatory children with cerebral palsy. Research in Developmental Disabilities,; 33(4): 1087-1094.

9. Hemmingsson $\mathrm{H}$, Borgestig $\mathrm{M}$. (2020) Usability of Eye-Gaze Controlled Computers in Sweden: A Total Population Survey. International Journal of Environmental Research and Public Health; 17(5): 1639.

10. Liljenquist, K., O’Neil, M. E., \& Bjornson, K. F. (2018):Utilization of Physical Therapy Services During Transition for Young People With Cerebral Palsy: A Call for Improved Care Into Adulthood. Physical Therapy; 98(9): 796-803.

11. Olajide A .(2013):.Psychological impact of cerebral palsy on families: The African perspective.J Neurosci Rural Pract.; 4(2): 159163. 